\title{
Predicting treatment resistance in schizophrenia patients
}

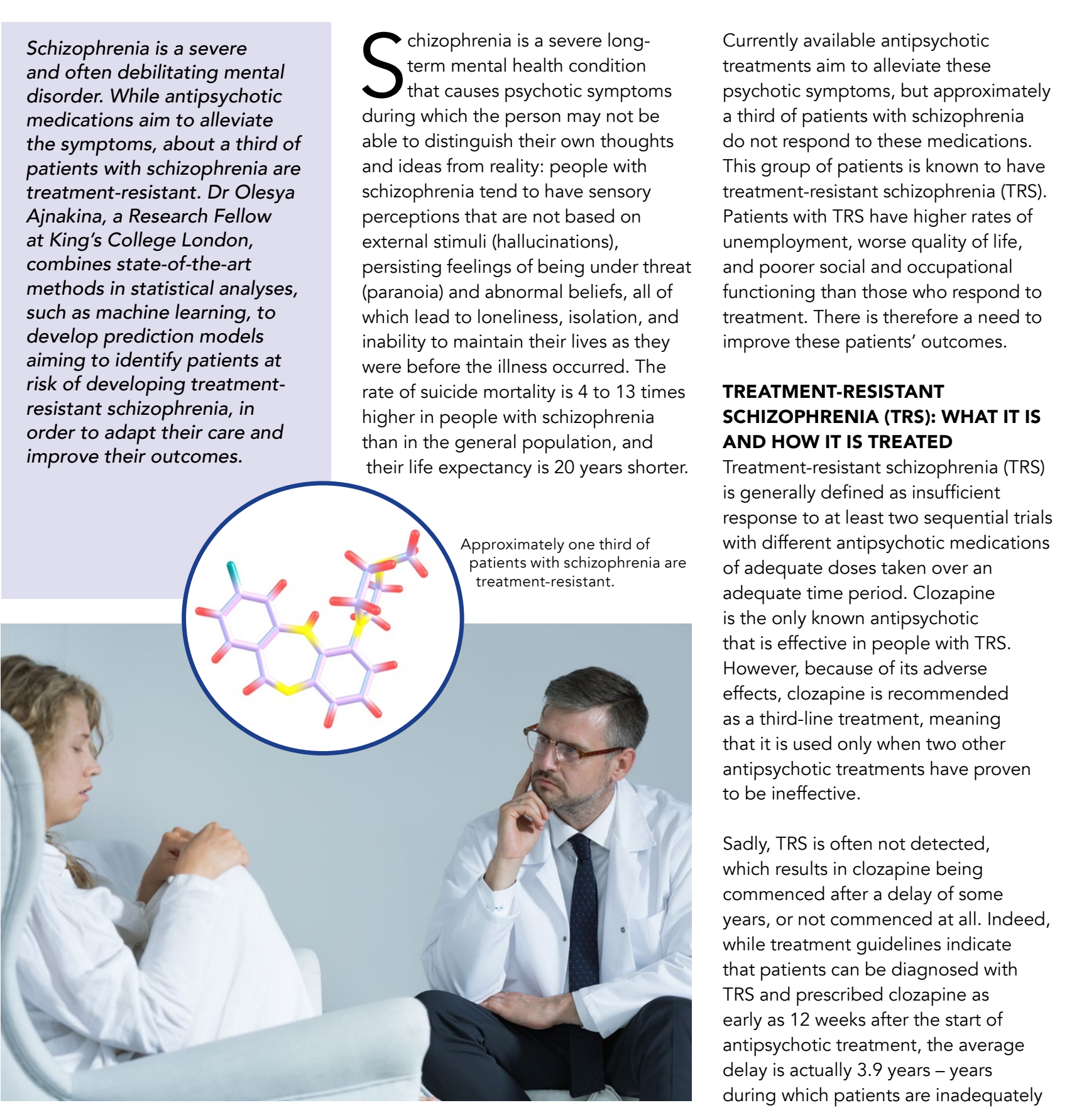

Treatment-resistant schizophrenia is not a single entity

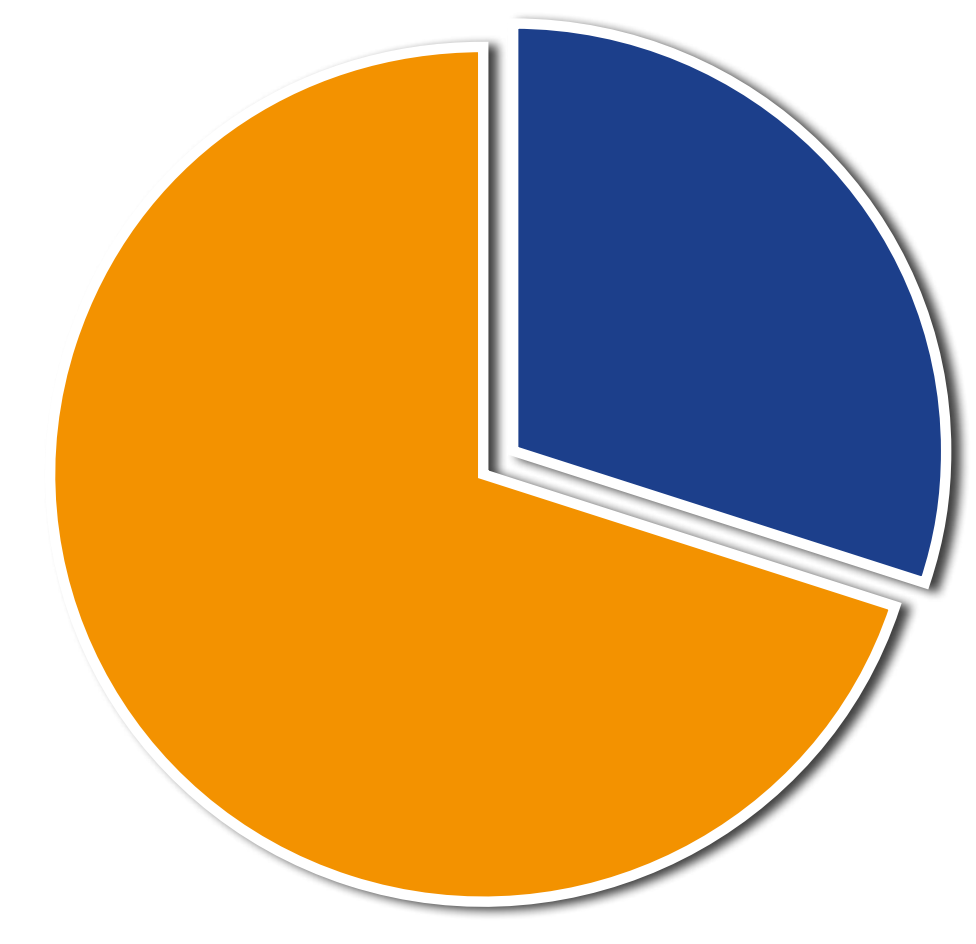

Late treatmen
schizophrenia

Early treatment-resistant (E-TR)

treated, consequently exposing them and their loved ones to a long period of unnecessary suffering. In fact, Dr Ajnakina and her team observed that over a period of five years, barely a half of patients with TRS commenced treatment with clozapine. The other haf did not commence it, despite meeting cheria for his treatment. Shortening the period of inadequate treatment would be beneficial

would be beneficial their loved ones, especially as a shorter delay is associated with a better response to

clozapine and subsequently improved quality of lives. A way to do that is to identify patients who are likely to develop TRS earlier they in the course of their illness and expe

TREATMENT-RESISTANT CHIZOPHRENIA IS NOT A INGLE ENTITY

TRS is a complex phenomenon; decades of researchers shed only a
limited light on our understanding sympto ver fist treatment for the resistant (E-TR) schizophrenia) whereas, the remaining $30 \%$ of TRS patients, broadly defined as late treatment-resistant (L-TR) schizoph gradually transition to treatment findings were ground-breaking as they
were the first to show that among of why it develops. The limited progress in research of TRS made might be due to the fact that TRS was being investigated as a single entity, which may not be entirely accurate. Indeed, Dr Ajnakina and her team were among the first researchers to demonstrate that treatment-resistant subtypes with differe ifferent subtypes with different underlying those patients with schizophrenia who will be treatment-resistant, a any treatments with non-clozapine antipsychotic medications from the very start of their treatment. Early treatment-resistant (E-TR) patients wil initially favourably respond to nonclozapine antipsychotic medications but gradually transition to treatment resistance; this group is known as late treatment-resistant (L-TR). The high proportion of patients who display E-TR highlights the importance of reducing the delay in clozapine use, as all these patients are inadequately treated for too long. In the meantime the existence of L-TR further complicates th detection of TRS, as may stop responding. may stop responding

\section{PREDICTORS: WHAT ARE THEY?} Identifying risk factors related to allows a rapid assessment of an individual risk for TRS subtypes, which is at the core of more in-depth risk assessment, follow-up monitoring and individually tailored prevention strategies. Predictors of TRS are risk factors indicating that a patient with schizophrenia is more at risk of being resistant to treatment. Identifying such predictors would be useful to identify TRS patents earler during treatment, so that they can be prescribed clozapine earler. Besides, predictors would also help scientists to understand

the causes of TRS. Different teams

A crucial benefit of prediction models $\begin{aligned} & \text { of researchers, } \\ & \text { including } D r \\ & \text { Ainakina's team }\end{aligned}$ is their ability to inform individuals of have tried to identify their risk of an illness.

around $70 \%$ of TRS patients did not respond to antipsychotic medications
from the very first treatment for their resistance having initially responded to antipsychotic medications. These or example, in a longitudinal study, Dr Ajnakina and her team reported that ted with clozapine were hose that, at the time of the first contact with healh services for schizophrenia, This finding severe psychopathology. This finding indicates that a clinician may be more likely to prescribe clozapine when the symptomatic presentation differis with TRS who conetwern patients with TRS who commenced clozapine 
that patients treated with clozapine were more likely to be living with fam the start of clozapin suggesting that depend on family support and stable living arrangements. Reports from oth teams indicate that various factors such as male gender, autumn or win birth, poor pre-morbid functioning and rural upbringing may also contribute to treatment resistance, although more data would be needed to confirm the role of these factors as predictors for TRS.

\section{PREDICTION MODELS AND WHY} THEY ARE IMPORTANT

A crucial benefit of prediction models is their abilty to inform individuals of their risk of an illness. For those who have developed a ill hess, predition modes trajectory Reliable prediction modes can thus guide clinicians' decisions to implement preventive interventions or personalised medicine strategies, to improve patients' outcomes.

To estimate even more precisely a patient's risk of developing treatment resistance, it is imperative to employ statistical learning algorithms that are recognised as optimal for personalised risk prediction. This is because they will enable the development of accurate prediction models for estimating individual, rather than average, risk for a disorder outcome during the illness, and clinical findings. These methods achieve this by, instead of looking at individual predictors one at a time, which significantly increases a risk of producing unrealisable results, simultaneously taking into account all the predictors while adjusting for any biases that may be present, such as correlated between the variables and multiple testing.

\section{PREDICTING TREATMENT}

RESISTANCE

Despite the vast personal and societal impact of schizophrenia, the preventive measures for people at risk of developing the disease, and personalised curative treatment options for people who have developed the illiness, were lacking. Therefore, Dr Ajnakina and her team are developing

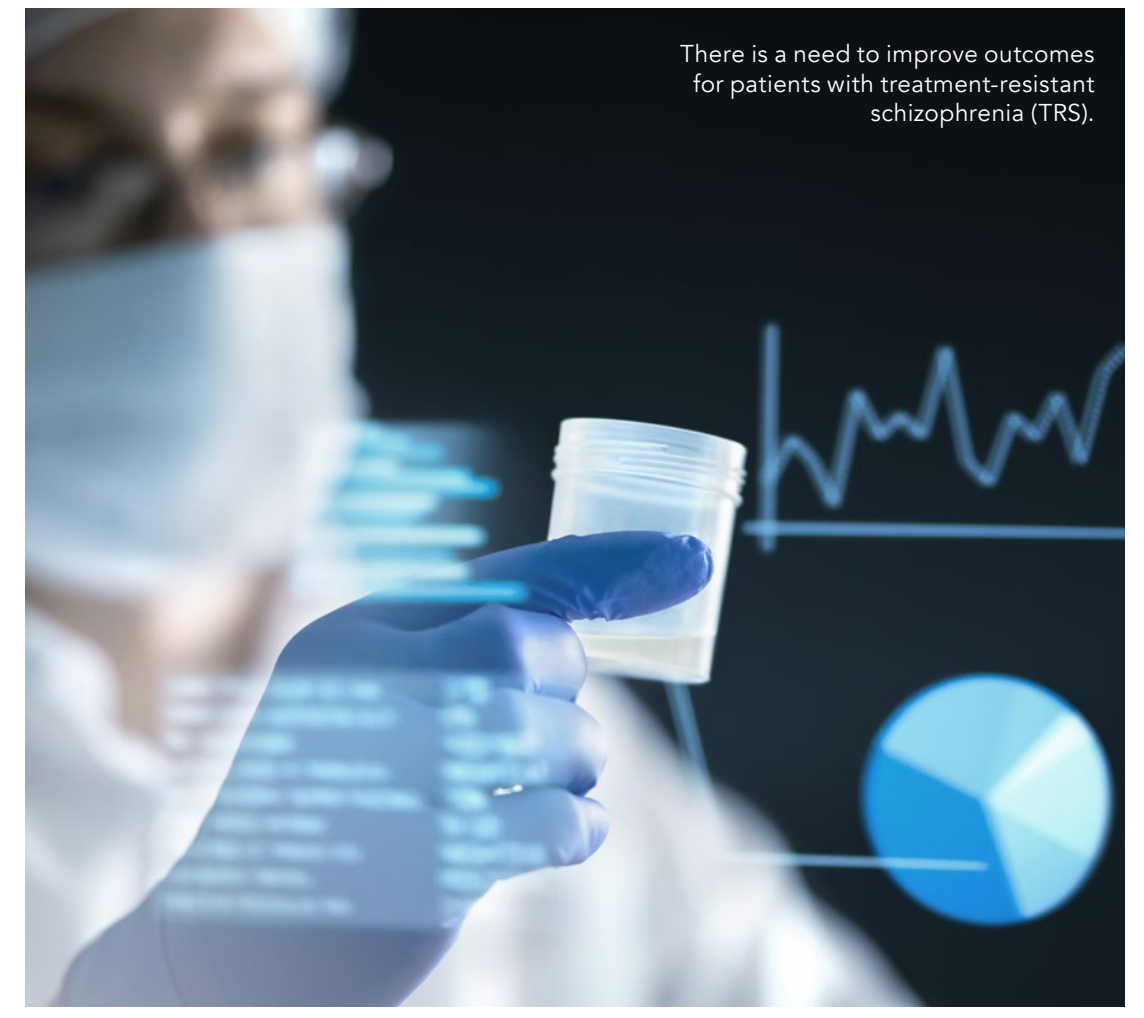

an individual risk for E-TR and L-TR, with the aim of understanding patients' risk of developing treatment resistance, response to the ongoing treatment with antipsychotic medications

Having focused on factors known to be associated with poor schizophrenia outcomes, such as adverse childhood expeninces, substance use and lack of

now included in clinical guidelines for therapeutic management. Moreover the developed prediction models developing E-TR and L-TR following onset of schizophrenia showed a strong ability to identify those schizophrenia patients who will not develop these TRS subtypes in the following 5 years, whereas, the high specificity implies these models are likely to

\section{For those who have developed an illness,} prediction models can estimate their most likely illness trajectory.

intensive statistical learning methods, particularly regularised regression methods, have been suggested as optimal methods for clinical and personalised risk prediction.

The developed models had good discriminative ability to identify those schizophrenia patients who were at a greater vs lesser risk to meet criteria discriminative ability for each model discriminative ability for each model was on par with prediction models for and cardiovascular dis, beast cancer schizophrenia patients as high risk for TRS subtypes in the following 5 years. Thus, utilising these models in practice diagnosed schizophrenia patients being exposed to inappropriate intervention plans designed for treatment resistant

Although further work is needed to improve them, these prediction models isk for TRS subtypes, which can be isseful to mplempen, which can be can reduce the chances for newly risk for TRS subssment of an individual

\section{Behind the Research}

Dr Olesya Ajnakina

\section{Research Objectives}

Dr Ajnakina's research career has focused on predicting who is at risk for developing psychosis includin cher patients. To achieve these goals, Dr Ainakina combines the start-of-the-art methods in statistical analyses, such as machine learning and interrogation of uman genomes.

\section{Detail}

Olesya Ajnakina

Department of Biostatistics and Health Informatics Institute of Psychiatry, Psychology and Neuroscience King's College London

PO20, 16 De Crespigny Park

London SE5 8AF, UK

Bio Medicine at King's College London. Dr Ajnakina holds PhD in predicting psychosis outcomes from King's College London where she was supervised by Sir Robin Murray. Prior to that Dr Ajnakina graduated in the top $5 \%$ of the BSc in Psychology class of 2011 and later pursued an MSc in Clinical Neuroscience (2012).

Funding

Fesearch

Collaborators

Sir Robin Murray who is Professor of Psychiatric Research at the Institute of Psychiatry, Psychology and Neuroscience (IoPPN), King's College London (KCL) and a Fellow of the Royal Society.

- Professor Daniel Stahl who is Professor in Medical Statistics and Statistical Learning at the Institute of Psychiatry, Psychology and Neuroscience (IoPPN), King's College London (KCL).

\section{NIHR}

NIHR $\mid \begin{aligned} & \text { National Institute } \\ & \text { for HealthResearch }\end{aligned}$
KING'S LONDON

\section{References}

- Lally, J., Ajnakina, O., Di Forti, M., Trotta, A., Demjaha, A., Kolliakou, A., Mondelli, V., Reis Marques, T., Parian MacCabe, J H, Gaughra, F. \& Murray, R. M. (2016) Two distinct patterns of treatment resistance: clinical predictors of treatment resistance in first-episode schizophrenia spectrum psychoses. Psychological Medicine, 46(15), 3231-3240. https://doi.org/10.1017/ S0033291716002014

A. P., Murray, R. M., \& MacCabe, J. H. (2019). Predictors of treatment resistant schizophrenia: a systematic review of prospective observational studies. Psychological Medicine, 1-10. Advance online publication. https://doi.org/10.1017/ S0033291719002083

- Ajnakina, O., Agbedjro, D., Lally, J., Forti, M. D., Trotta A., Mondelli, V., Pariante, C., Dazzan, P., Gaughran, F., Fisher, H. L., David, A., Murray, R. M., \& Stahl, D. 2020). Predic firg onset of early- Rnd late-treatment using advanced shrinkage statistical methods in a small sample. Psychiatry Research, 294, 113527. Advance online publication. https://doi.org/10.1016/j. psychres.2020.113527

- Steyerberg, E. W., \& Vergouwe, Y. (2014). Towards better clinical prediction models: seven steps for development and an $\mathrm{ABCD}$ for validation. European Heart Journal, 35(29), 1925-1931. https://doi.org/10.1093/eurhearti/ehu207

\section{Personal Response}

What was a challenge that you faced while conducting this research and how did you overcome it?

II When researchers and clinicians talk about treatment resistance in schizophrenia, they often, unintentionally affected patients and their loved one. This is not the case! The challenging part of this work was to provid a balance between the severity of this iliness and reached an era when with the right tools, we can create personalised risk prediction tools for schizophrenia that would lead to better intervention and prevention strategies. I dealt with this challenge by critically caution for overoptimism. Yes, our work still needs to be improved by using a larger sample, but the first step is
the hardest, and we certainly did it in this work. 